\title{
Conocimientos, actitudes y prácticas de familias de adolescentes con discapacidad cognitiva en sexualidad y afectividad*
}

\section{Knowledge, attitudes and practices of families of teenagers with cognitive disabilities in sexuality and affectivity}

\author{
Mauricio Polanco Valenzuela ** \\ ORCID 0000-0002-6225-5020 \\ Universidad Internacional Iberoamericana, \\ UNINI, México \\ Juan Luis Martín Ayala \\ Universidad Internacional Iberoamericana, \\ UNINI, México \\ Recibido: 16 de abril de 2017 \\ Revisado: 2 de mayo de 2017 \\ Aceptado: 23 de junio de 2017
}

\section{Resumen}

Este artículo parte de la reflexión acerca de la vulneración de derechos de las personas con discapacidad, al desconocer que la sexualidad y afectividad también son fundamentales en sus vidas y configuran el ejercicio de los derechos sexuales y reproductivos; sin embargo, se han logrado importantes avances para su reconocimiento como sujetos titulares de derechos y generado múltiples normas que reivindican su titularidad y garantía; no obstante, estudios adelantados en Colombia y en el mundo, evidencian la persistencia de barreras fundamentadas en el desconocimiento, discriminación y falsas creencias sobre dichos aspectos de las personas con discapacidad. El interés del estudio fue indagar mediante una encuesta, los conocimientos, actitudes y prácticas de padres, madres y cuidadores de adolescentes con discapacidad cognitiva de una institución educativa especializada de Bogotá, para que los resultados contribuyan a fortalecer capacidades de las familias y de instituciones con acciones pedagógicas que fomenten la garantía de derechos y el mejoramiento de la calidad de vida de esta población.

Palabras clave: discapacidad cognitiva, sexualidad, afectividad, derechos sexuales y reproductivos, familia.

* $\quad$ Artículo de investigación. DOI: http://dx.doi.org/10.15332/s1794-9998.2017.0002.04

** Correspondencia: Mauricio Polanco. Universidad Internacional Iberoamericana / UNINI México. Dirección postal: Calle 106 No. $54-93$ (Consultorio 505). Barrio Puente Largo, Bogotá, Colombia. Correo electrónico: mauriciopolanco@hotmail.com 


\section{Abstract}

This article starts from the reflection that there is violation of the rights of people with disabilities, not knowing that sexuality and affectivity are also fundamental in their lives and make up the exercise of Sexual and Reproductive Rights; however, significant progress has been achieved for its recognition as rights holders and generated multiple norms that claim for ownership and guarantee of them; nevertheless, advanced studies in Colombia and in the world, evidence the persistence of barriers based on lack of awareness, discrimination and false beliefs about these aspects of people with disabilities. The interest of the study was to investigate through a survey by the knowledge, attitudes and practices of parents and caregivers of teenagers with cognitive disabilities of a specialized educational institution of Bogotá so that the results contribute to strengthen the abilities of the families and the institutions with pedagogical actions that promote the guarantee of rights and the improvement of the quality of life of this population.

Key words: disabilities cognitive, sexuality, affectivity, sexual and reproductive rights, family

\section{Introducción}

Las personas con discapacidad sufren múltiples vulneraciones a sus derechos por parte de otras personas no discapacitadas, profesionales e instituciones. Se les limita o excluye de la participación social, en contradicción con los principios establecidos en la Convención de Derechos de las Personas con Discapacidad (CDPCD), impulsada por la Organización de las Naciones Unidas (ONU). Con relación a la salud sexual y reproductiva (SSR) preocupa ampliamente el cómo dichas personas se ven enfrentadas histórica y constantemente a barreras físicas, sociales, educativas, culturales y económicas que impiden la garantía y el ejercicio de sus derechos en igualdad de condiciones con las demás personas (Carrillo, 2012; ONU, 2006; Parra, 2013; Trujillo, Cuervo y Escobar, 2001; Vargas, 2006). Parte de estas barreras corresponde a bajos conocimientos en temas de sexualidad, actitudes muchas veces discriminatorias y prácticas familiares que cuestionan las capacidades de las personas con discapacidad e impiden el ejercicio pleno de la SSR (González, 2005; Ministerio de Educación Nacional, 2007; Navarro, 2000; Torices, 2009). Para los adolescentes con discapacidad cognitiva esto se traduce en evidentes dificultades para vivir de manera adecuada, adaptarse apropiadamente al medio en el que se desenvuelven, y les impacta negativamente en la formación de su identidad personal, la autoestima y el ejercicio satisfactorio de su sexualidad (Cobo, 2012; Cáceres, 2014; De la Cruz y Cabezón, 2006).
Según el Informe Mundial sobre la Discapacidad (Organización Mundial de la Salud y Banco Mundial, 2011), se calcula que aproximadamente mil millones de personas $-15 \%$ de la población mundial-, viven con algún tipo de discapacidad. Cifra que sumada a la situación que padecen países en conflicto como Colombia, podría alcanzar hasta un $18 \%$ de la población total. Teniendo en cuenta las últimas proyecciones del Departamento Administrativo Nacional de Estadística del país (DANE) (2007), la población en Colombia ubicada entre los 10 y 19 años para el 2017 es de 4'185.511 personas, entre mujeres y hombres, lo cual representa el 8,49\% de la población total del país (49'291.609), cifra que en el contexto general es alta, de la cual se estima que un segmento importante lo constituye la población con discapacidad. Según el Instituto Colombiano de Bienestar Familiar (ICBF) (2013) de 56.474 niños, niñas y adolescentes registrados con discapacidad, el $58.3 \%$ son hombres y el $47.7 \%$ son mujeres; el $49 \%$ tiene entre 15 y 18 años y el 32,7 \% entre 11 y 14 años. De ellos, el 95,1\% pertenece a estratos socioeconómicos bajos y el $4,9 \%$ a los más altos. Los porcentajes presentados mundial y nacionalmente dan cuenta de la necesidad de continuar trabajando de manera vigorosa con las personas con discapacidad y sus familias en todos los aspectos relacionados con sus vidas.

Sin embargo para contrarrestar lo anterior, las barreras y los altos porcentajes de población con discapacidad, la normatividad internacional y nacional han logrado importantes avances al reconocer 
desde los años setenta el concepto de derechos humanos de las personas con discapacidad, hecho reafirmado durante la Conferencia Mundial sobre Derechos Humanos en Viena en 1993 y materializado en 2006 con la Convención, fundamental: "para promover, proteger y salvaguardar el goce pleno y en condiciones de igualdad de todos los derechos humanos y libertades fundamentales de todas las personas con discapacidad, promoviendo el respeto de su dignidad inherente" (ONU, 2006, p.4). La Convención, además de reconocer su capacidad jurídica en igualdad de condiciones y la obligatoriedad de protegerles en el seno del hogar como fuera de él, contra la explotación, violencia y abuso, establece la garantía y el acceso a programas educativos y de SSR. En Colombia, con la entrada en vigencia de la Constitución Política de 1991, la obligatoriedad se hace evidente al quedar señalado en el Artículo 13 que: “el Estado protegerá especialmente a aquellas personas que por su condición económica, física o mental, se encuentren en circunstancia de debilidad manifiesta y sancionará los abusos o maltratos que contra ellas se cometan". Así mismo, el tema empieza a ser considerado y estudiado con mayor fuerza por la academia y las entidades públicas y privadas de los diferentes sectores, lo que lleva a tomar conciencia y fortalecer el desarrollo legislativo que ha contribuido a su incorporación en las agendas políticas y sociales del país (Alcaldía de Bogotá, 2012). A pesar de ello, los conceptos que se manejan no son los más precisos, se adopta el goce efectivo de los derechos pero no de manera uniforme, se plantean necesidades educativas especiales más no la educación inclusiva, se expresa como personas afectadas por discapacidad y no personas con discapacidad, se plantea el enfoque de integración no de inclusión y se incorpora un enfoque asistencialista no el de derechos como sí lo afirma la Convención (Parra, 2013). Al respecto se puede mencionar como el logro jurisprudencial colombiano más reciente, el pronunciamiento que sobre los DSR de las personas con discapacidad adelantó la Corte Constitucional mediante la Sentencia T-573 de 2016, en consonancia con la Convención, el enfoque de derechos y el modelo social de discapacidad.

Al igual que la normatividad, el concepto de discapacidad ha evolucionado en el tiempo, con modelos que establecen marcos de actuación propios frente a las personas con discapacidad. Se ha pasado de concepciones que sostienen que el origen de la discapacidad es por motivos religiosos, científicos o derivados por una serie de limitaciones individuales, o por causas, ni religiosas ni científicas, sino en gran medida, sociales (Palacios, 2008). Este último hace referencia al modelo social, asumido en América Latina y el país, adoptado plenamente por el presente estudio al reflejar un cambio de paradigma, que centra el eje de la discapacidad no en la persona y sus carencias, sino en su interacción con el entorno y las dificultades que este le presenta para participar plenamente. Lo que significa que las personas con discapacidad aportan a la sociedad en igual medida que el resto de las personas y se responde de forma más adecuada a sus necesidades e inquietudes: "propiciando la inclusión social y sentándose sobre la base de determinados principios: vida independiente, no discriminación, accesibilidad universal, normalización del entorno, diálogo civil, entre otros" (Palacios, 2008, p.27). Al respecto vale la pena retomar lo que la Clasificación Internacional del Funcionamiento de la Discapacidad y la Salud (CIF) estableció en 2001, y es que el problema del término y su utilización peyorativa va más allá del lenguaje de acuerdo a las actitudes de los individuos y de la sociedad, pues la discapacidad existirá independientemente de cómo se la denomine (Carrillo, 2012). De allí que lo más importante de la clasificación es que engloba en su totalidad las características fundamentales de las personas con discapacidad y lo señalado en la normatividad: capacidades, entorno y funcionamiento (Muntaner, 2010).

De acuerdo a lo anterior y con relación a la sexualidad, toda persona vive, expresa y le da significado a esta dimensión en función del conjunto de normas, creencias y prácticas sociales, que influyen significativamente en su comportamiento. La sexualidad es un aspecto fundamental del ser humano, presente a lo largo de su vida; por ello experimenta sensaciones, emociones, sentimientos, afectos y deseos; abarca a su vez al sexo, las identidades, los roles de género, el erotismo, el placer, la intimidad, la reproducción y la orientación sexual; puede incluir todas estas dimensiones, no obstante, no todas ellas se vivencian o expresan siempre (OMS, 2011). 
Por tales razones se deduce que el desarrollo sexual de las personas con discapacidad difiere muy poco del de las llamadas "normales". El interés por el sexo suele ser precoz como en cualquier otro niño, sus características sexuales secundarias suelen aparecer también durante la adolescencia, los cambios en el comportamiento e interacción con el sexo opuesto es similar, así como el incremento generalizado en su interés por la sexualidad (Navarro, 2000). La exclusión, discriminación, incomprensión y falta de tolerancia para con las personas con discapacidad han sido impuestas por el mismo ser humano y por la cultura en que se desarrolla, lo que conduce en muchos casos a la vulneración de los DSR, ya que al ser percibidos como poco normales, se sitúan en lugares desventajosos de la sociedad, se exponen a la discriminación y al abuso, se estereotipan y estigmatizan, se les califica de eternos niños, se les acusa de tener una conducta sexual exacerbada, se les niega una educación al respecto para evitar "incitarles" su sexualidad, se les controla la reproductividad por temor a que engendren "más discapacitados" y por tanto se les niega la posibilidad de ejercer la maternidad o paternidad, se les considera incapaces para captar abusos de los que pueden ser objeto o porque prevalece la opinión respecto a que las personas con discapacidad cognitiva no deben o están en capacidad de disfrutar de cada uno de ellos (González, 2005; Torices, 2009; Universidad del Rosario, 2015).

A ello se agrega lo que otros autores como Garvía y Miquel (2009) señalan al respecto y es que la sexualidad al componerse al menos de tres funciones: reproductora, placentera y afectivo-relacional, en el caso de las personas con discapacidad, preocupa en mayor medida la función reproductora dependiendo del tipo de discapacidad padecida, por las implicaciones físicas y emocionales en las etapas de gestación y posterior crianza de sus hijos, en tanto que las otras dos funciones aunque se asocian con sentimientos de vergüenza, ansiedad, temor, problemas, como juicio empobrecido, razonamiento deficiente para establecer relaciones afectivas, muestras de afecto indiscriminado, dificultades de expresión de sentimientos y falta de intimidad. Sin embargo, es importante identificar cuál es la verdadera y real necesidad que esconden algunas presiones o manifestaciones de la persona con discapacidad, puesto que estaría una necesidad de relación afectiva y no sexual como se podría malinterpretar (Caricote, 2012).

Estas falsas creencias, actitudes erróneas y conocimientos limitados en el tema no son propios solamente de un contexto social particular sino también del sistema familiar que forma parte de él, en donde se trasmiten e instauran valores, principios y creencias respecto a todos los aspectos de la vida del ser humano. La influencia familiar es fundamental desde los primeros años y para las personas con discapacidad se constituye en uno de sus soportes básicos, ya que lo que experimente en su propia sexualidad depende en gran medida de lo que ha aprendido y vivenciado a lo largo de su ciclo vital, es decir, el rol familiar sobre esos aspectos marcará de manera significativa su sexualidad y su vida. Ser padre o madre es una tarea difícil especialmente cuando se trata de manejar situaciones complejas de las cuales se tiene poca o ninguna información, lo que se complejiza aún más cuando se tiene un hijo con discapacidad, de allí su importancia como guía y soporte social (Hernández, López y Ávila, 2006). Al existir un integrante con alguna discapacidad, la familia puede verse afectada en sus roles, dinámica y en lo interpersonal, por tanto el desafío y las tareas para esta son mayores. Cuando en una familia un miembro nace o adquiere una discapacidad, la comunicación se hace dolorosa e inesperada, lo que lleva a experimentar otros sentimientos de angustia, depresión, culpa y aislamiento social (Caricote, 2012). Situación que se apoya muchas veces sobre la base de una interacción rígida y sin cambios, gira en torno a la persona con discapacidad y cuyas relaciones afectivas internas y externas están mediatizadas por esta. Por el contrario, para Amor Pan (2000) existen familias que contando con una persona con discapacidad cognitiva logran equilibrio con normas más flexibles para adecuarse a distintas situaciones, realizar actividades creativas y satisfacer necesidades de todos. Esto es importante porque así como lo afirma López (2011), familias que apoyan el desarrollo afectivo-sexual de sus hijos, se constituyen en adecuadas figuras de apego y en modelos aceptables, protegen de riesgos reales e imaginarios, responden a sucesos evolutivos, ofrecen y buscan ayudas, colaboran con 
la escuela y aprenden a desvincularse sin dañar ni maltratar. Toda formación en sexualidad y afectividad va más allá de la persona con discapacidad, abarca su círculo cercano y otras redes de apoyo y se sustenta en el enfoque de derechos humanos.

Con base en lo anterior, surge la necesidad de adelantar un estudio que indague sobre los conocimientos, actitudes y prácticas que tienen las familias y cuidadores de adolescentes con discapacidad cognitiva en los aspectos relacionados con su sexualidad y afectividad, por tratarse como se ha visto, de áreas fundamentales para el crecimiento integral de todo ser humano y cuyo desarrollo es básico para el fortalecimiento personal, familiar, social y de salud. Este estudio resulta pertinente por el impacto personal, familiar y cultural que tiene el profundizar sobre la sexualidad y la afectividad, por las herramientas teórico-prácticas que se derivan de sus hallazgos, por la importancia que tiene la discapacidad en el mundo y en el país, por el amplio respaldo normativo y legal con que cuenta, y fundamentalmente porque el marco de derechos debe soportar el accionar de los profesionales de las áreas educativas, de salud y de protección.

\section{Método}

Como el estudio pretende esencialmente interpretar acciones, significados y hechos relevantes situados en un contexto social específico como es el de la discapacidad, la investigación corresponde a un estudio cualitativo, transversal, de tipo observacional descriptivo, en la medida en que se detallan e interpretan los conocimientos, actitudes y prácticas que tienen padres y madres respecto a la sexualidad y la afectividad de los adolescentes con discapacidad cognitiva (Trillos, 2008). Por tanto se utilizó una encuesta que permitió, además, puntualizar la frecuencia y distribución con que se presentan brechas en información y conductas mediadas por mitos, creencias y prejuicios en sexualidad y afectividad.

\section{Muestra}

La población objeto de estudio estuvo constituida por 32 familias de adolescentes entre los 12 y 19 años de edad, hombres y mujeres, con discapacidad cognitiva según lo establecido en la Clasificación Internacional del Funcionamiento de la Discapacidad y la Salud (CIF), que forman parte de una institución educativa con amplia experiencia en ayudar a niños, niñas y adolescentes con discapacidad cognitiva, cuyo modelo de enseñanza-aprendizaje está basado en explorar sus mayores fortalezas y afianzar sus habilidades y talentos; para ello trabaja desde el grado preescolar hasta terminar la básica secundaria, y apoya adicionalmente la integración al trabajo. La institución se encuentra ubicada en un municipio cercano a la ciudad de Bogotá y aunque muchos de sus estudiantes residen en la capital, los demás provienen de familias de muy bajos recursos de la zona y de otras poblaciones. Dado que la investigación es cualitativa y no busca hacer inferencia estadística, el muestreo fue no probabilístico por conveniencia, en donde las unidades de muestreo siguieron criterios de conveniencia del investigador y de los objetivos de la investigación -riqueza de información por tener hijos adolescentes con discapacidad cognitiva, conveniente accesibilidad a la institución, proximidad, disposición para participar en el estudio, principalmente-, y fueron seleccionadas secuencialmente hasta lograr el punto de representatividad en la diversidad en las estructuras socio-económicas, culturales y espaciales requeridas para ilustrar los conocimientos, actitudes y prácticas de padres, madres y cuidadores. Las familias fueron seleccionadas conjuntamente con la institución por el contacto directo que tienen con ellas, previa consulta y aceptación voluntaria.

\section{Procedimiento}

La información se recolectó a través de fuentes secundarias correspondientes a la literatura técnico-científica relacionada con el objeto de estudio y de fuentes primarias con padres, madres y cuidadores de adolescentes con discapacidad cognitiva y con profesionales que intervienen a esta población. Los instrumentos utilizados fueron entrevistas semi-estructuradas y una encuesta de conocimientos, actitudes y prácticas (CAP). Con ella se valoró el grado de conocimientos, actitudes y prácticas de las familias con relación a las dimensiones de sexualidad y afectividad, mediante 
categorías y variables netamente cualitativas, ordinales y nominales en algunos casos, como: conocimientos: nivel de conciencia y comprensión que se tienen respecto a los derechos, normas y recursos de protección, sexualidad, afectividad, riesgos, fuentes de información; actitudes: para indagar por el sentir y la manera de comportarse frente a la sexualidad y afectividad de los adolescentes con discapacidad: mitos, creencias y estereotipos, aprobación de la expresión sexual y afectiva, estigma o discriminación; prácticas, para evaluar el cómo actúan o aplican los conocimientos con sus hijos adolescentes: expresiones o manifestaciones, acciones frente a su expresión, dinámica familiar, emociones y sentimientos en la familia, responsabilidades/funciones familiares.

Con el propósito de garantizar la validez del estudio y evitar un sesgo de subjetividad, se incluyeron respuestas del tipo de selección múltiple y escalas tipo Likert para medir las actitudes asumidas y conocer el grado de conformidad del encuestado con las afirmaciones propuestas en una escala que medía desde "totalmente de acuerdo" hasta "totalmente en desacuerdo". La encuesta fue revisada y validada por pares expertos y por un grupo representativo de la población objeto de estudio, con quien se adelantó una prueba piloto que permitió realizar ajustes y precisiones en cuanto a comprensión, suficiencia y extensión. Para responder a la validez y aumentar la confianza en la autenticidad de los datos y en su interpretación, la información obtenida se trianguló para comparar y verificar su consistencia a partir de otras fuentes como encuestas a actores clave, observaciones y documentos técnicos.

El procesamiento de los datos se realizó con estadística descriptiva, se elaboraron tablas de frecuencias relativas y absolutas para las variables incluidas en la encuesta. Para almacenar y procesar la información cuantitativa se creó una estructura de la base de datos en hojas de cálculo y en módulo de vista del programa estadístico SPSS, de acuerdo con su definición operativa de medición y su operacionalización según escala de medición, tipo de variables y relación entre estas. En cada etapa se garantizó la privacidad, la confidencialidad con cuestionario anónimo y voluntario, la aceptación y consentimiento informado y la evitación de cualquier tipo de riesgo para los participantes.

\section{Resultados}

Los hallazgos de la encuesta CAP proporcionan información básica sobre los vacíos en cuanto a conocimientos y actitudes que predominan frente a la asunción de la sexualidad y afectividad, y prácticas más comunes de las familias con adolescentes con discapacidad cognitiva y que coinciden con hallazgos de investigaciones previas. En la tabla 1 se presenta la caracterización socio-demográfica de las 32 personas que participaron en el estudio.

Tabla 1.

Caracterización sociodemográfica de la población encuestada

\begin{tabular}{|c|c|c|c|c|c|c|c|c|c|c|c|c|c|c|c|c|c|c|c|c|c|c|}
\hline \multirow{3}{*}{$\begin{array}{l}\text { Parentesco } \\
\text { con el ado- } \\
\text { lescente }\end{array}$} & \multicolumn{10}{|c|}{ Nivel educativo } & \multicolumn{4}{|c|}{ Procedencia } & \multicolumn{8}{|c|}{ Estado civil } \\
\hline & \multicolumn{2}{|c|}{$\begin{array}{l}\text { Secunda- } \\
\text { ria }\end{array}$} & \multicolumn{2}{|c|}{ Técnica } & \multicolumn{2}{|c|}{$\begin{array}{l}\text { Tecnoló- } \\
\text { gica }\end{array}$} & \multicolumn{2}{|c|}{$\begin{array}{l}\text { Universi- } \\
\text { taria }\end{array}$} & \multicolumn{2}{|c|}{$\begin{array}{l}\text { Postgra- } \\
\text { do }\end{array}$} & \multicolumn{2}{|c|}{ Rural } & \multicolumn{2}{|c|}{ Urbana } & \multicolumn{2}{|c|}{ Casado } & \multicolumn{2}{|c|}{$\begin{array}{c}\text { Divor- } \\
\text { ciado/ } \\
\text { Separado }\end{array}$} & \multicolumn{2}{|c|}{ Soltero } & \multicolumn{2}{|c|}{$\begin{array}{l}\text { Unión } \\
\text { Libre }\end{array}$} \\
\hline & $\mathrm{FR}$ & $\%$ & FR & $\%$ & $\mathrm{FR}$ & $\%$ & $\mathrm{FR}$ & $\%$ & FR & $\%$ & FR & $\%$ & FR & $\%$ & FR & $\%$ & FR & $\%$ & FR & $\%$ & $\mathrm{FR}$ & $\%$ \\
\hline Abuela & 1 & 3,13 & 0 & 0 & 0 & 0 & 0 & 0 & 0 & 0 & 0 & 0 & 1 & 3,13 & 1 & 3,13 & 0 & 0 & 0 & 0 & 0 & 0 \\
\hline Madre & 3 & 9,38 & 4 & 12,5 & 2 & 6,25 & 9 & 28,13 & 6 & 18,8 & 3 & 9,38 & 21 & 65,6 & 14 & 43,8 & 7 & 21,88 & 1 & 3,13 & 2 & 6,25 \\
\hline Padre & 1 & 3,13 & 0 & 0 & 0 & 0 & 4 & 12,5 & 2 & 6,25 & 0 & 0 & 7 & 21,9 & 6 & 18,8 & 1 & 3,13 & 0 & 0 & 0 & 0 \\
\hline $\begin{array}{l}\text { Total } \\
\text { general }\end{array}$ & 5 & 15,63 & 4 & 12,5 & 2 & 6,25 & 13 & 40,63 & 8 & 25 & 3 & 9,38 & 29 & 90,6 & 21 & 65,63 & 8 & 25 & 1 & 3,13 & 2 & 6,25 \\
\hline
\end{tabular}

Fuente: elaboración propia. 


\section{Conocimientos}

Centró su atención sobre la información que maneja la familia acerca de DSR, normatividad y recursos que protegen a la población con discapacidad, nivel de conciencia y comprensión que tienen sobre la sexualidad, la afectividad y los riesgos. De este modo, se halló que el $96 \%$ de padres y madres tienen claridad sobre derechos humanos y en DSR; el $41 \%$ desconoce que en el país haya normas y leyes para la protección de los derechos de personas con discapacidad. Con relación al conocimiento sobre los recursos del sector de protección, el $37 \%$ desconoce la existencia de instituciones que trabajan con dicha población. En conocimientos específicos se encontró que en nociones básicas sobre sexualidad, riesgos y protección en SSR, un elevado porcentaje (entre $63 \%$ y $94 \%$ ) posee la información suficiente; no obstante, frente a género y orientación sexual, se presenta confusión. Así mismo, el manejo es básico en violencias de género, tipologías y en violencia sexual. De igual modo, el $22 \%$ no identifica correctamente las condiciones en las que se despenalizó el aborto en Colombia. Con relación a los DSR, padres y madres (90\%) manifiestan reconocer que las personas con discapacidad pueden tener relaciones sexuales 0 que tienen derecho a ser respetados en la toma de decisiones respecto a su sexualidad (88\%). Un $53 \%$ no tiene claridad sobre el concepto de interdicción. Frente a conocimientos puntuales sobre afectividad y vínculos, el $84 \%$ identifica las emociones básicas y sus diversas expresiones, y el $91 \%$ reconoce que en el país existen diferentes tipos de conformación familiar. Padres y madres registran que la mejor y más completa información sobre sexualidad no se obtiene en el colegio y que internet y las redes sociales transmiten información errada sobre sexualidad. La tabla 2 presenta las frecuencias relativas y absolutas obtenidas en conocimientos.

\section{Actitudes}

La valoración acerca de las actitudes se hizo a través de una escala tipo Likert que indagaba por el sentir y por la manera de actuar de padres y madres frente a la sexualidad y afectividad de sus hijos con discapacidad cognitiva, con el propósito de evidenciar el grado de asunción de mitos, creencias, estereotipos, aprobación de la expresión sexual y afectiva, estigma y discriminación. Así, se encontró que un 6 $\%$ de los padres y madres piensa que la sexualidad de sus hijos en esta condición es exagerada y un 22 $\%$ mostró neutralidad; un $9 \%$ está de acuerdo con la creencia de que los adolescentes con discapacidad cognitiva son asexuados o que tienen una sexualidad infantilizada. Sin embargo se debe considerar que la mitad de los encuestados (entre $50 \%$ y $59 \%$ ), no está en desacuerdo con dichas creencias. Un $40 \%$ de los padres y madres estiman que los adolescentes no tienen autonomía frente a su sexualidad, pero un 85 \% sí está de acuerdo con que tienen capacidad para establecer relaciones de pareja. Frente a la posibilidad de que puedan tener hijos, las respuestas estuvieron dispersas entre un $44 \%$ que está de acuerdo, un $25 \%$ que no sabe realmente - neutro-y un $31 \%$ que señaló no estar de acuerdo. Respecto de las creencias y estereotipos en sexualidad y reproducción, el $50 \%$ considera no estar de acuerdo en que puedan tener relaciones sexuales sin amor y un $9 \%$ muestra neutralidad; no obstante, un $94 \%$ sí mostró acuerdo con que los adolescentes experimentan deseo sexual y un $69 \%$ está de acuerdo con que tienen derecho a regular su fertilidad. Llama la atención que un $19 \%$ de padres y madres no están de acuerdo en que sus hijos tengan la capacidad de procrear. El grado de asunción de las actitudes se aprecia en la tabla 3, presentada a continuación.

Al indagar sobre las creencias relacionadas con la maternidad y/o paternidad de los adolescentes, se encontró que el $28,1 \%$ de padres y madres piensa que se deben esterilizar y el $12,5 \%$, regular. Frente a la orientación sexual diversa, el 68,8 \% de padres y madres creen que se debe aceptar; sin embargo, un 3,1\%, rechazar. Con relación a lo que piensan acerca de las expresiones de afectividad y sexualidad, el $90,6 \%$ de padres y madres las comprende cuando son demostraciones de afecto, pero al ser expresiones de su sexualidad, ese mismo porcentaje cree que es mejor orientarlo; un 3,1\% piensa que se debe acudir al castigo. Respecto a lo que la familia concibe como la mejor forma para enseñar sobre sexualidad, el $59,4 \%$ piensa que orientando y el 34,4 \% apoyándose de expertos. En cuanto a temas de afectividad, $43,8 \%$ manifiesta que orientar es clave y un $34,4 \%$, con el ejemplo. Solamente el $26 \%$ de la familia piensa que la sociedad acepta la sexualidad y que el $74 \%$ la rechaza y discrimina. 
Tabla 2.

Evaluación de conocimientos

N. ${ }^{\circ}$

Enunciado

Verdadero Falso

NS/ TOTAL

1 Los derechos del niño son derechos humanos que buscan proteger a los niños como los seres Total $\quad 30 \quad 32 \quad 0 \quad 32$

1 humanos que son y se constituyen en garantías fundamentales para su bienestar y desarrollo $\quad \% \quad \begin{array}{lllll}94 \% & 6 \% & 0 \% & 100 \%\end{array}$

Los derechos sexuales y reproductivos son los derechos humanos, internacionalmente recono-Total $\quad 26 \quad 2 \quad 4 \quad 32$

2 cidos, que garantizan el desarrollo libre, sano, seguro y satisfactorio de la vida sexual, repro-- $\frac{1}{8} \quad 81 \% \quad 6 \% \quad 13 \% \quad 100 \%$ ductiva y de la convivencia sexual.

La convención sobre las personas con discapacidad es un instrumento de derechos humanos Total

3 con una dimensión explícita de desarrollo social. En ella se adopta una amplia clasificación

de las personas con discapacidad y se afirma que debe gozar de todos los derechos humanos $\quad \begin{array}{lllll}6 & 69 \% & 13 \% & 19 \% & 100 \%\end{array}$

$y$ libertades fundamentales

4 En Colombia existen normas y leyes para la protección de los derechos de las personas con Total 19

discapacidad

$\% \quad 59 \%$

5 En el País y el Bogotá concretamente existen instituciones públicas o privadas que trabajan Total

con adolescentes con discapacidad y sus familias

$\% \quad 63 \%$

6 La sexualidad como dimensión fundamental de todos los seres humanos, cumple una función Total 22

placentera

7 Las categoría sexo y genero significan y representan lo mismo para los seres humanos

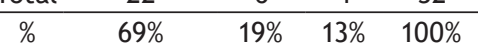

\begin{tabular}{ccccc} 
Total & 19 & 10 & 3 & 32 \\
\hline $59 \%$ & $31 \%$ & $9 \%$ & $100 \%$
\end{tabular}

8 La identidad sexual o de genero es una vivencia interna e individual que puede corresponder o Total

no con el sexo asignado al nacer. En nuestra sociedad existen muchas formas de identidad sexual $\%$

9 La orientación sexual es una atracción emocional, romántica, sexual o afectiva duradera Total 20 hacia otros

10 Los métodos anticonceptivos se emplean para prevenir el embarazo no deseado

11 El condón o preservativo previene el embarazo y protege de infecciones de transmisión sexual $\frac{\text { Tota }}{2}$

12 Los adolescentes con discapacidad cognitiva no pueden tener relaciones sexuales

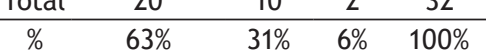

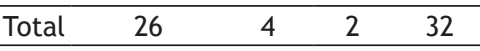

\begin{tabular}{lllll}
$\%$ & $81 \%$ & $13 \%$ & $6 \%$ & $100 \%$ \\
\hline
\end{tabular}

Total 30

$\% \quad 94 \%$

13 Las caricias, los besos, y las palabras cariñosa se consideran como expresiones y manifesta-

13 ciones afectivas

\begin{tabular}{ccccc} 
Total & 3 & 29 & 0 & 32 \\
\hline$\%$ & $9 \%$ & $91 \%$ & $0 \%$ & $100 \%$
\end{tabular}

En nuestro País existen diferentes formas de organización familiar o tipos de familia, como por Total

14 ejemplo la nuclear (conformada por padre, madre, e hijos) o el madre solterísimo (mujeres que son madres pero que no tienen conyuge)

15 Los adolecentes con discapacidad cognitiva tienen derecho a ser respetados en la toma de Total

15 decisiones con respecto a su sexualidad

Total 32

Las cinco emociones básicas del ser humano son la alegría, el amor, la tristeza, la rabia y el Tota miedo

17 La violencia de genero es toda conducta que tenga como fin el menoscabo de la dignidad, el Total 23

daño a ala integridad física o la violación de derechos de un hombre por el mero hecho de serlo \% $72 \%$

18 La violencia física, emociona, sexual y patrimonial hacen parte de las violencias de género \begin{tabular}{cccccc} 
Total & 28 & 1 & 3 & 32 \\
\cline { 2 - 6 } & $\%$ & $88 \%$ & $3 \%$ & $9 \%$ & $100 \%$
\end{tabular}

En la violencia sexual están presentes todas las relacione so actos no consentidos por la per-Total $\quad 31 \quad 32 \quad 1 \quad 32$

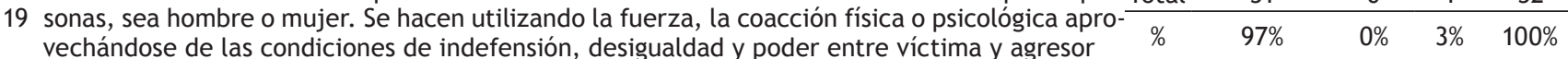

20 En abuso sexual hace referencia a todas aquellas frases y conductas dirigidas a menoscabar la Total 26

20 libertad y la dignidad mediante expresiones machistas, sexistas o des obligantes

\begin{tabular}{|c|c|c|}
\hline$\frac{26}{81 \%}$ & $\begin{array}{c}4 \\
13 \%\end{array}$ & 2 \\
\hline
\end{tabular}

21 Las infecciones de transmisión sexual o ITS son aquellas que se transmites de una personas a Tot

21 otra que está sana, por contacto sexual

En Colombia se despenalizó el aborto en tres condiciones: Cuando peligra la vida o la salud Total 25

22 de la mujer, cuando se presenta malformación del feto incompatible con al vida y cuando el embarazo es producto de abuso, violación, incesto, transferencia de ovulo o inseminación no consentida

23 La interdicción de una personas con discapacidad tiene que ver con la privación de sus dere-Total

23 chos civiles por parte de una autoridad judicial

24 La mejor y más completa información sobre sexualidad es la que se da en las clases de los Tota

colegios

\begin{tabular}{ccccc}
\hline Total & 29 & 3 & 0 & 32 \\
\hline$\%$ & $91 \%$ & $9 \%$ & $0 \%$ & $100 \%$ \\
\hline
\end{tabular}

El internet y las redes sociales como el facebook transmiten información errada sobre sexua-Total

25 lidad

$\begin{array}{llll}\% & 78 \% & 6 \% & 100 \%\end{array}$

\begin{tabular}{ccccc} 
Total & 15 & 8 & 9 & 32 \\
\hline & $47 \%$ & $25 \%$ & $28 \%$ & $100 \%$ \\
\hline
\end{tabular}

\begin{tabular}{ccccc}
\hline Total & 2 & 29 & 1 & 32 \\
\hline$\%$ & $6 \%$ & $91 \%$ & $3 \%$ & $100 \%$ \\
\hline
\end{tabular}

Total 28

$\% \quad 88 \%$


Tabla 3.

Grado de asunción de mitos, creencias y estereotipos

\begin{tabular}{|c|c|c|c|c|c|c|c|c|c|c|c|c|c|}
\hline \multirow[t]{2}{*}{ n. ${ }^{\circ}$} & \multirow[t]{2}{*}{ Actitud } & \multicolumn{2}{|c|}{$\begin{array}{l}\text { En total } \\
\text { acuerdo }\end{array}$} & \multicolumn{2}{|c|}{ Acuerdo } & \multicolumn{2}{|c|}{$\begin{array}{l}\text { Ni acuerdo } \\
\text { ni des- } \\
\text { acuerdo }\end{array}$} & \multicolumn{2}{|c|}{ Desacuerdo } & \multicolumn{2}{|c|}{$\begin{array}{c}\text { En total } \\
\text { desacuerdo }\end{array}$} & \multicolumn{2}{|c|}{ NS/NR } \\
\hline & & FR & $\%$ & FR & $\%$ & FR & $\%$ & FR & $\%$ & FR & $\%$ & FR & $\%$ \\
\hline 1 & $\begin{array}{l}\text { Los adolescentes con discapacidad } \\
\text { cognitiva tienen una sexualidad } \\
\text { exagerada }\end{array}$ & 0 & 0 & 2 & 6,25 & 7 & 21,88 & 8 & 25,00 & 15 & 46,88 & 0 & 0 \\
\hline 2 & $\begin{array}{l}\text { Los adolescentes con discapacidad } \\
\text { cognitiva son asexuados }\end{array}$ & 0 & 0 & 3 & 9,38 & 3 & 9,38 & 7 & 21,88 & 19 & 59,38 & 0 & 0 \\
\hline 3 & $\begin{array}{l}\text { La sexualidad de los adolescentes con } \\
\text { discapacidad cognitiva es muy infantil }\end{array}$ & 3 & 9,38 & 3 & 9,38 & 10 & 31,25 & 9 & 28,13 & 7 & 21,88 & 0 & 0 \\
\hline 4 & $\begin{array}{l}\text { Los adolescentes con discapacidad } \\
\text { cognitiva tienen autonomía frente a su } \\
\text { sexualidad }\end{array}$ & 2 & 6,25 & 11 & 34,38 & 5 & 15,63 & 10 & 31,25 & 3 & 9,38 & 1 & 3,13 \\
\hline 5 & $\begin{array}{l}\text { Los adolescentes con discapacidad } \\
\text { cognitiva tienen la capacidad de } \\
\text { establecer relaciones de pareja }\end{array}$ & 12 & 37,5 & 15 & 46,88 & 3 & 9,38 & 0 & 0,00 & 2 & 6,25 & 0 & 0 \\
\hline 6 & $\begin{array}{l}\text { Los adolescentes con discapacidad } \\
\text { cognitiva pueden tener hijos }\end{array}$ & 6 & 18,75 & 8 & 25 & 8 & 25,00 & 5 & 15,63 & 3 & 9,38 & 2 & 6,25 \\
\hline 7 & $\begin{array}{l}\text { Los adolescentes con discapacidad } \\
\text { cognitiva podría tener relaciones sexuales } \\
\text { sin amor }\end{array}$ & 5 & 15,63 & 7 & 21,88 & 3 & 9,38 & 7 & 21,88 & 9 & 28,13 & 1 & 3,13 \\
\hline 8 & $\begin{array}{l}\text { Los adolescentes con discapacidad } \\
\text { cognitiva experimentan deseo sexual }\end{array}$ & 20 & 62,5 & 10 & 31,25 & 2 & 6,25 & 0 & 0 & 0 & 0 & 0 & 0 \\
\hline 9 & $\begin{array}{l}\text { Los adolescentes con discapacidad } \\
\text { cognitiva tienen derecho a regular si } \\
\text { fertilidad }\end{array}$ & 13 & 40,63 & 9 & 28,13 & 4 & 12,50 & 2 & 6,25 & 3 & 1 & 1 & 3,13 \\
\hline 10 & $\begin{array}{l}\text { Los hijos adolescentes con discapacidad } \\
\text { cognitiva están en capacidad de quedar en } \\
\text { embarazo o dejar embarazada a alguien }\end{array}$ & 11 & 34,38 & 12 & 37,5 & 3 & 9,38 & 2 & 6,25 & 4 & 0 & 0 & 0 \\
\hline
\end{tabular}

Fuente: elaboración propia.

\section{Prácticas}

Se evaluaron con preguntas cerradas que indagaban por la forma en que se manifiestan o aplican los conocimientos de padres y madres sobre expresiones de la sexualidad y la afectividad, emociones y sentimientos, responsabilidades y funciones. Las expresiones más comunes de la afectividad reconocidas por su familia son los abrazos $(65,6 \%$ ) y las palabras cariñosas (37,5\%), en tanto que las manifestaciones de la sexualidad se reconocen como caricias $(34,4 \%)$ y besos $(28,1 \%)$; sin embargo hay otras expresiones que son similares en lo afectivo y lo sexual. Con respecto a manifestaciones físicas o verbales, la familia tiende en mayor proporción a reaccionar brindándoles orientación y siendo compresivos. No obstante, como se ve en la tabla 4, en algunos casos como cuando el/la adolescente tiene prácticas autoeróticas o expresa malas palabras, se presentan reacciones negativas como regaño, castigo o rechazo.

Al indagar por la manera en cómo educan a sus hijos, se halló que las familias utilizan prácticas orientadoras en un $59,4 \%$ y democráticas en un 25 $\%$; sin embargo, 3 (9,4 \%) señalaron que son permisivos. Con relación a las normas y límites que emplean, indicaron en un $37,5 \%$ que son orientadoras, pero de igual modo hay prácticas opuestas en igual proporción: 22 \% reconocen que son autoritarios y otro tanto que son democráticas.

En el mismo sentido, el 78,1\% de padres y madres reconocen que normas y límites con que educan a sus hijos son claras, un $12,5 \%$ rígidas y un $6,3 \%$, difusas. En cuanto a la comunicación, el $84,4 \%$ de ellos aceptan que esta es positiva y abierta, mientras un $12,5 \%$ reservada y fría. 
Tabla 4.

Expresiones de afectividad y sexualidad de los adolescentes con discapacidad cognitiva

\begin{tabular}{lcccccccccc}
\hline \multicolumn{2}{c}{ Reacciones frente a las manifestaciones físicas o verbales del adolescente con discapacidad cognitiva } \\
\hline Manifestación & Autoerotismo & $\begin{array}{c}\text { Lenguaje ina- } \\
\text { propiado }\end{array}$ & $\begin{array}{c}\text { Expresiones } \\
\text { afectivas (be- } \\
\text { sos, caricias, } \\
\text { abrazos) }\end{array}$ & $\begin{array}{c}\text { Relaciones inter- } \\
\text { personales que } \\
\text { establece (amis- } \\
\text { tad, noviazgo) }\end{array}$ & $\begin{array}{c}\text { Manejo de sus } \\
\text { emociones (ira, } \\
\text { amor, miedo, } \\
\text { tristeza, alegria) }\end{array}$ \\
\hline \multicolumn{1}{c}{ Reacción } & FR & $\%$ & FR & $\%$ & FR & $\%$ & FR & $\%$ & FR & $\%$ \\
\hline Castiga físicamente & 0 & $0,0 \%$ & 1 & $3,1 \%$ & 0 & $0,0 \%$ & 0 & $0,0 \%$ & 0 & $0,0 \%$ \\
\hline Regaña & 3 & $9,4 \%$ & 9 & $28,1 \%$ & 0 & $0,0 \%$ & 0 & $0,0 \%$ & 0 & $0,0 \%$ \\
\hline Orienta o educa & 21 & $65,6 \%$ & 19 & $59,4 \%$ & 17 & $53,1 \%$ & 19 & $59,4 \%$ & 23 & $71,9 \%$ \\
\hline Comprende & 4 & $12,5 \%$ & 2 & $6,3 \%$ & 12 & $37,5 \%$ & 14 & $43,8 \%$ & 11 & $34,4 \%$ \\
\hline Es indiferente & 1 & $3,1 \%$ & 0 & $0,0 \%$ & 0 & $0,0 \%$ & 1 & $3,1 \%$ & 0 & $0,0 \%$ \\
\hline Cupabiliza & 0 & $0,0 \%$ & 0 & $0,0 \%$ & 0 & $0,0 \%$ & 0 & $0,0 \%$ & 0 & $0,0 \%$ \\
\hline Rechaza & 1 & $3,1 \%$ & 2 & $6,3 \%$ & 0 & $0,0 \%$ & 0 & $0,0 \%$ & 0 & $0,0 \%$ \\
\hline No lo hace & 1 & $3,1 \%$ & 1 & $3,1 \%$ & 1 & $3,1 \%$ & 0 & $0,0 \%$ & 0 & $0,0 \%$ \\
\hline
\end{tabular}

Fuente: elaboración propia.

\section{Discusión}

El entorno familiar y el contexto comunitario del grupo de adolescentes con discapacidad cognitiva objeto del estudio, estuvo representado por familias con estructura nuclear, en su mayoría por mujeres (madres/cuidadoras), que poseen estudios superiores, proceden de sectores urbanos, casadas y con una edad promedio de 50 años, lo que presupone que los adolescentes están creciendo en hogares que cuentan con conocimientos, habilidades y competencias suficientes para orientar y atender sus necesidades en sexualidad y afectividad. El entorno socio económico de las familias y el no presentar ninguna condición de vulnerabilidad satisface las necesidades básicas y favorece el acceso de los adolescentes a servicios educativos, de salud, culturales, entre otros, que protegen y garantizan sus derechos, dado que los encuestados son económicamente activos y en su mayoría de estrato medio. La tercera parte de las familias encuestadas participa activamente en espacios sociales o culturales, lo que posibilita el contar con otras redes de apoyo diferentes a la familia e instituciones educativas, además de brindar oportunidades de interacción de los adolescentes con discapacidad con pares.

Hay un conocimiento significativo sobre derechos humanos y particularmente sobre DSR, así como los vínculos entre sexualidad y afectividad, que determinan la importancia de reforzarlos. Se requiere llenar un vacío en conocimientos sobre normatividad para evitar vulnerar derechos, así como en la divulgación de los servicios y su prestación para esta población, para que les ayude en la tarea de formación. A pesar de que cuentan con información específica de conceptos básicos sobre la sexualidad, riesgos y protección, hay algunos temas con mayor desconocimiento: género, orientación sexual, interrupción voluntaria del embarazo y violencias de género. Igualmente temen que sus hijos sean víctimas de violencia y por eso conocen más del tema. Las familias reconocen que los adolescentes pueden tener relaciones sexuales y tomar decisiones sobre su sexualidad; sin embargo no hay claridad del concepto de interdicción, de allí la importancia de sensibilizarlos en estos aspectos legales. A las familias encuestadas les parece insuficientes los conocimientos que reciben como comunidad educativa en los colegios al igual que la que obtienen a través de los medios de comunicación como las redes sociales y el internet. Por el contrario sienten que es un vacío que hay que llenar e incluso aprender a utilizarlos de forma crítica y educativa, porque las familias no se encuentran lo suficientemente preparadas para responder a las diversas inquietudes y demandas que suscitan sus adolescentes en términos de la expresión y vivencia de su sexualidad. A pesar de que en la mayoría de las personas existe un nivel de conocimientos aceptable persisten en algunos padres y madres mitos, 
creencias erróneas y estereotipos sobre la sexualidad y afectividad que limitan el ejercicio pleno y libre de sus DSR, al considerar que la expresión de sus hijos en esta área es un comportamiento inapropiado. Esto puede traducirse, como lo señala Cobo (2012) en una deficiente socialización de lo sexual y afectivo, soledad en lo emocional y lo social o en baja autoestima, o como lo corrobora Torices (1997) en una dependencia física y afectiva de los demás, en incapacidad para establecer vínculos afectivos con personas de igual o diferente condición, lo que afecta la manera de enfrentarse al mundo.

Padres y madres no reconocen la capacidad, así sea limitada, que tienen sus hijos para tomar decisiones autónomas respecto a la expresión de su sexualidad. Por el contrario se aprecia desconocimiento o negación de los DSR, como en la conformación de pareja, tener o no hijos, manejo del cuerpo, expresión de emociones y orientación sexual. Tampoco muestran avances en reconocer la sexualidad como una dimensión fundamental del ser humano. Las actitudes son contradictorias y ambivalentes en muchos casos, lo que podría llegar a causar vulneración en los DSR de sus hijos: regulación de la fertilidad, procreación y elección libre de pareja, porque persisten mitos que como lo señala Torices (2009), la persona con discapacidad podría engendrar otra persona con discapacidad y por ello hay que controlar su fertilidad. Las familias aun cuando son comprensivas frente a las expresiones afectivas -caricias, besos, abrazos y palabras cariñosas-, experimentan temores y resistencias a expresiones de la sexualidad, lo que se confirma cuando creen que sus adolescentes no deben tener relaciones sexuales sin amor o que hay que castigarlos por ello. En cuanto a la afectividad, padres y madres están de acuerdo en que a sus hijos hay que brindarles orientación y ejemplo mientras que para la sexualidad, además de orientación, se requiere apoyo de expertos. Esto concuerda con lo hallado en vacíos de conocimiento y en la falta de preparación para el acompañamiento. Pese a la percepción de mayor aceptación por parte de la sociedad, padres y madres consideran que hay una alta discriminación y estigmatización que se refleja en actitudes de rechazo, negación, burla, denigración, menosprecio e incomprensión, lo que va en contravía de los avances en materia de inclusión y equidad de las personas con discapacidad. Por los resultados hallados en cuanto al reconocimiento de las expresiones o manifestaciones sexuales y afectivas de adolescentes, se denota cierto grado de confusión al no haber claridad sobre la connotación que unas u otras tienen para el adolescente, porque hay manifestaciones físicas como las caricias y los besos que pueden ser tomadas bien como una expresión de afecto o por el contrario con contenido sexual. Por tal motivo, la crianza de adolescentes con discapacidad implica una reacomodación en la dinámica familiar y en su interior, a la forma en que se reconocen o censuran conductas percibidas como inapropiadas.

El estudio permite observar la falta de preparación para asumir y orientar las manifestaciones físicas o verbales, al persistir prácticas como el castigo, rechazo, regaño o indiferencia que mengua la autonomía de los adolescentes y constriñen su desarrollo emocional, social y sexual. Para autores como Cobo (2012) lo anterior se relaciona con la deficiente socialización sexual, debido principalmente a la negación de la sexualidad y la represión de cualquier forma de manifestación erótica, cuando lo que se precisa es justamente la orientación en ello, tal como lo manifiestan al abordar la manera en que educan a sus hijos. Tanto para la educación como para la imposición de límites y normas, las familias entienden que sí cumplen un rol orientador; sin embargo, no todos reconocen esta función y ceden a prácticas poco favorables para el desarrollo de sus hijos, basadas en la permisividad o autoritarismo. Esto contradice lo hallado por Amor Pan (2000), quien manifiesta que si una familia con algún miembro con discapacidad cognitiva logra un equilibrio con normas flexibles, se le facilitará el adecuarse a distintas situaciones y a seguir realizando actividades creativas de su vida. Finalmente, los resultados permiten deducir que en el marco de la discapacidad cognitiva y sus familias, la comunicación en cuanto a la sexualidad y la afectividad es un elemento clave para la educación y el desarrollo integral de los adolescentes, que aunque es favorable, aún persisten prácticas no expresivas y distantes con sus hijos con consecuencias negativas para ambos. 


\section{Conclusiones}

A partir del análisis de conocimientos, actitudes y prácticas que las familias tienen con respecto a los adolescentes con discapacidad cognitiva, la comunidad científica y los tomadores de decisión podrían disponer de referentes más claros y ajustados a dicha realidad, lo que garantizaría una mayor inclusión social, el respeto por los derechos humanos y los DSR, la cualificación para la atención en salud y la mejor prestación de servicios. De igual manera, se propicia la formación de profesionales de sectores educativos, de salud o protección, en aquéllos aspectos relacionados con la sexualidad, la afectividad y la discapacidad, y a la comprensión de las familias. Esta posibilidad se constituye en una oportunidad para mejorar condiciones de vida de la comunidad y una respuesta más a problemas sociales que les afectan.

De los hallazgos obtenidos, el estudio se constituye en un campo propicio para trabajar con diversos tipos de discapacidad y con diferentes poblaciones que se encuentran en riesgo o en situación de vulnerabilidad, así como la necesidad de indagar y comprender en profundidad lo que se presenta en otros contextos sociales y regiones. Principios como el de derechos humanos, salud sexual y reproductiva, enfoque diferencial, educación inclusiva y perspectiva de género, deben ser parte de los programas, proyectos y estrategias de formación de los agentes educativos, acompañados de material didáctico y recursos humanos especializados. Por todo ello se requiere superar las resistencias que pueda generar la cuestión planteada, sensibilizar en mayor número a las familias, los profesionales y las instituciones públicas y privadas sobre la importancia que tienen los DSR para la población con discapacidad, vencer los temores y angustias que representa enfrentar la sexualidad de los adolescentes con discapacidad cognitiva y, finalmente, canalizar cada vez mayores recursos humanos y físicos que fortalezcan las familias y las instituciones vinculadas estrechamente con el tema. Esto incluye la posibilidad de ofrecer capacitación permanente e intencional a las familias y a los profesionales relacionados con la discapacidad, al diseñarse y validarse un modelo pedagógico que abarque todos los ejes transversales que preocupan y que se evidencian con el estudio, lo que se constituye en una oportunidad más de mejoramiento de las condiciones de vida de las comunidades y de respuesta a problemas sociales que afectan a la población.

\section{Referencias}

Alcaldía Mayor de Bogotá. (2012). Proyecto de atención integral a personas con discapacidad, familias, cuidadores y cuidadoras - Cerrando brechas. Bogotá: SDIS.

Amor Pan, J.R. (2000). Afectividad y sexualidad en la persona con deficiencia intelectual. España: Ed. Pontificio de Comillas.

Cáceres, C.M. (2014). La educación afectivo-sexual en las personas con discapacidad intelectual y su relación con el trabajo social. España: Universidad de la Laguna.

Caricote, E. (2012). La sexualidad en la discapacidad intelectual. Ensayo Educere, 16, 395-402.

Carrillo, M. (2012). Inteligencia, conducta adaptativa y calidad de vida. Interacciones explicativas de la discapacidad intelectual y la intervención optimizadora. Barcelona: Universidad Autónoma de Barcelona.

Cobo, C. (Coord.). (2012). Protocolo sobre relaciones interpersonales y sexualidad en personas con discapacidad intelectual, trastornos del Aspectro Autista y otras discapacidades con déficit cognitivo usuarias de centros residenciales. Recuperado de http://www.juntadeandalucia.es.

Constitución Política de Colombia. (1991). Constitución Política de Colombia. Recuperado de http://www. constitucioncolombia.com/

Corte Constitucional de Colombia. (19 de octubre de 2016). Sentencia T-573. [MS. Luis Ernesto Vargas Silva] Recuperado de https://www.minsalud. gov.co/proteccionsocial/promocion-social/ Discapacidad/Paginas/convocatoria-sentencia-t573.aspx 
Departamento Administrativo Nacional de Estadística. (DANE. (2007). Proyecciones de población 20052020. Recuperado de https://www.dane.gov.co/ files/investigaciones/poblacion/proyepobla06_ 20/8Tablasvida1985_2020.pdf.

De la Cruz, C. y Cabezón, O. (2006). Apuntes de Educación Sexual. Sobre la sexualidad de los niños y niñas con discapacidad. Recuperado de http://www.juntadeandalucia.es.

Garvía, B. y Miquel, M.J. (2009). La vida sexual y afectiva de las personas con síndrome de Down. España: FCSD.

González, M.N. (2005). La sexualidad del adolescente con discapacidad y su abordaje en el ámbito familiar. Revista Pensamiento Actual, 5, 55-60.

Hernández, L., López, I. y Ávila, G. (2006). Participación de la familia para rehabilitar sexualmente a la persona con discapacidad. Aula Abierta, 87, 3-26.

Instituto Colombiano de Bienestar Familiar. (2013). Informe. Recuperado de http://www.icbf.gov.co.

López, F. (2011). Guía para el desarrollo de la afectividad y de la sexualidad en personas con discapacidad intelectual. Junta de Castilla y León: $\mathrm{dDC}$

Ministerio de Educación Nacional. (2007). Guía para el docente: Educación sexual de niños, niñas y jóvenes con discapacidad intelectual. Chile.

Muntaner. J. (2010). La persona con retraso mental, bases para su inclusión social y educativa: Guía para padres y educadores. Málaga: Ediciones Aljibe.

Navarro, J.I. (2000). Desarrollo afectivo-sexual de la persona con discapacidad psíquica. Sexualidad y personas con discapacidad psíquica, 1, 66.

Organización de las Naciones Unidas (ONU). (2006). Convención Internacional de Naciones Unidas sobre los Derechos de las Personas con Discapacidad. Recuperado de http://www. un.org/esa/socdev/enable/documents/tccconvs.pdf.

Organización Mundial de la Salud (OMS). (2011). Informe Mundial sobre la Discapacidad. Resumen. Recuperado de http://www. who.int/disabilities/world_report/2011/ summary_es.pdf.

Palacios, A. (2008). El modelo social de discapacidad: orígenes, caracterización y plasmación en la Convención Internacional sobre los Derechos de las Personas con Discapacidad. Madrid: CERMI.

Parra, C. (2013). Estructura institucional de la discapacidad en el Estado colombiano. Civilizar. Ciencias Sociales y Humanas, 13, 79-102.

Torices, I. (1997). La sexualidad en las personas con discapacidad. México: Universidad Autónoma de Puebla.

Torices, I. (2009). La sexualidad y discapacidad física. México: Editorial Trillas.

Trillos, C.E. (2008). Conceptos básicos en epidemiología, epidemiología básica y principios de investigación. Medellín: CIB.

Trujillo, A., Cuervo, C. y Escobar, M. (2001). Documento de trabajo. Contexto: discapacidad e inclusión social. Bogotá: Universidad Nacional de Colombia.

Universidad del Rosario. (2015). Derechos sexuales y reproductivos de las personas con discapacidad intelectual: ¿Está preparada nuestra sociedad? Bogotá: Universidad del Rosario, Escuela de Medicina y Ciencias de la Salud.

Vargas P., D.R. (2006). Representaciones sociales de la discapacidad por parte de los miembros de los consejos locales de discapacidad de la ciudad de Bogotá. Bogotá: Universidad Nacional. 